NOTE TECHNIQUE

\title{
REVUE DES MÉTHODES D'ESTIMATION DE L'EFFECTIF D'UNE POPULATION PAR PÊCHES SUCCESSIVES AVEC RETRAIT PROGRAMME D'ESTIMATION D'EFFECTIF PAR LA METTHODE DE CARLE ET STRUB
}

\section{GERDEAUX}

INRA - Station d'Hydrobiologie Lacustre B.P. 11 F - 74203 THONON-LES-BAINS Cedex, France

Reçu le 4 Août 1986

Accepté le 23 Janvier 1987

Received 4 August, 1986 Accepted 23 January, 1987

\section{RÉSUME}

Les méthodes traditionnelles de LESLIE, de DE LURY sont rappelées. La méthode de ZIPPIN est présentée avec la possibilité de réaliser les calculs par ordinateur sans recours aux estimations graphiques. La méthode de CARLE et STRUB étant plus robuste, un programme de calcul sur microordinateur est proposé en annexe.

\section{REVIEW OF METHODS OF ESTIMATING POPULATION SIZE BASED ON REMOVAL DATA COMPUTER PROGRAM OF SIZE ESTIMATION WITH THE CARLE AND STRUB METHOD}

\section{ABSTRACT}

The paper describes methods of estimating population size based on survey removal data. The Zippin maximum likelihood model is presented with the possibility of calculation with microcomputer. The Carle et Strub method is considered most convenient and a microcomputer program is published.

\section{INTRODUCTION}

Les méthodes d'estimation des effectifs des populations par pêches successives avec retrait des individus pêchés ne sont praticables que dans les milieux restreints faciles à pêcher ou parfois dans des pêcheries quand le prélèvement réduit de façon appréciable le stock présent (RICKER, 1975).

L'origine de ces méthodes remonte à HELLAND (1913-1914) qui estime l'effectif d'une population d'ours norvégiens à partir de statistiques de chasse. Depuis, ces méthodes ont fait l'objet de nombreux. développements mathématiques. Les premières développées sont des méthodes de régression (LESLIE et DAVIS, 1939 ; DE LURY, 1947). Les recherches d'estimateurs meilleurs ont été ensuite faites par la méthode du maximum de vraisemblance (ZIPPIN, 1956 et 1958), puis du maximum de vraisemblance pondéré (CARLE et STRUB, 1978). Une revue de ces méthodes a été publiée par COWX (1983). Dans cet article, l'auteur utilise des approximations et présente des méthodes dérivées de la méthode de ZIPPIN pour certains cas particuliers (JUNGE et LIBOSVARSKY, 1965 ; SEBER et LE CREN, 1967: ROBSON et REGIER, 1968). L’emploi actuel des microordinateurs facilite les calculs imposés par les méthodes modernes et rend inutiles les approximations. Bien que facile à programmer, un logiciel de calcul pour la méthode de CARLE et STRUB est présenté en annexe et on pourra se reporter à une publication de HIGGINS (1985) pour la méthode de ZIPPIN.

Notre propos est donc de rappeler les méthodes couramment employées en estimation des populations et d'introduire la méthode de CARLE et STRUB qui a l'avantage d'admettre une probabilité de capture variable.

\section{LES MÉTHODES D'ESTIMATION}

\section{1) Exemple}

Nous illustrerons chaque méthode par les données du cas concret d'une pêche électrique dans un secteur de $190 \mathrm{~m}$ de long, $728 \mathrm{~m}^{2}$ de surface, délimité par barrages de filets sur la rivière le Redon, affluent du lac Léman, le 9 novembre 1983. Dans ce secteur, le Redon est une petite rivière de $3,8 \mathrm{~m}$ de largeur moyenne, de $1.9 \%$ de pente, à fond caillouteux sans herbier et avec peu de caches profondes. Les résultats de la pêche électrique, lors des 3 passages successifs sont présentés dans le tableau 1 pour les truites fario de 10 a $15 \mathrm{~cm}$ et les truites de moins de $10 \mathrm{~cm}$ de longueur totale. 


\begin{tabular}{|l|c|c|}
\hline & $\begin{array}{c}\text { Truites fario } \\
10 \text { à } 15 \mathrm{~cm}\end{array}$ & $\begin{array}{c}\text { Truites fario } \\
\text { 1e passage }\end{array}$ \\
\hline 2e passage & 101 & $40 \mathrm{~cm}$ \\
\hline 3e passage & 12 & 18 \\
\hline Total & 2 & 7 \\
\hline
\end{tabular}

Tableau 1 : Résultats de 3 pêches électriques successives dans un secteur du Redon.

Table 1 : Number of trout in 3 successive catches from the Redon.

\section{2) Notation}

La notation suivante sera utilisée dans tous les calculs :

No $=$ taille de la population étudiée

$\mathrm{Ci}=$ nombre de poissons capturés à la pêche $\mathrm{i}$

$\mathrm{Ni}=$ nombre total de poissons capturés avant la pêche $i, c$ 'est-à-dire, l'effectif cumulé des i-1 premières pêches

$T=$ total des poissons capturés pour toutes les pêches

$k$ = nombre de pêches

$p=$ probabilité de capture d'un poisson durant une pêche

$q=1-p$

\section{3) Les hypothèses de travail}

Les hypothèses suivantes devraient être vérifiées pour que les méthodes d'estimation soient satisfaisantes.

a) La population doit être "stationnaire": il n'y a pas de natalité, pas de mortalité ; pas d'émigration et pas d'immigration. Dans le cas des pêches électriques pratiquées dans les rivières:

- on minimisera l'incidence du recrutement ou des mortalités en réalisant des pêches sur une période courte:

- on évitera le biais occasionné par les migrations en fermant le secteur par des filets barrages. L'effectif de la population inventoriée pourra ainsi être supposé constant.

b) La probabilité de capture est supposée être la même pour tous les individus pendant une pêche. En particulier, la taille du poisson ne doit pas influer sur sa capturabilité.

c) La probabilité de capture doit être constante d'une pêche à l'autre. Dans l'estimation de CARLE et STRUB nous verrons que la constance de cette probabilité n'est pas obligatoire fla probabilité p suivant elle-même une loi de probabilité). 


\section{4) Les méthodes de régression}

\section{a) La méthode de LESLIE}

L'effectif de la ième pêche est égal à la probabilité de capture multipliée par l'effectif de la population présente avant la ième pêche.

$$
\begin{aligned}
& \mathrm{Ci}=\left(\mathrm{No}_{\mathrm{o}}-\mathrm{Ni}\right) \mathrm{p} \\
& \mathrm{Ci}=-\mathrm{pNi}+\mathrm{pNo}
\end{aligned}
$$

Cette équation (1) montre que la relation entre la capture à l'instant i et la capture cumulative $\mathrm{Ni}$ avant cette pêche est une droite. Pour $\mathrm{Ci}=0$, nous obtenons $\mathrm{pNi}=\mathrm{pNo} c^{\prime}$ est-à-dire que la droite coupe l'axe des abscisses au point No et que la pente de la droite est une estimation de $p$. Le point d'intersection sur l'axe des $Y$ est le produit de la population initiale No et de la probabilité de capture.

On peut connaître graphiquement la valeur de No ou bien réaliser un calcul de régression linéaire simple pour obtenir l'équation de la droite et l'abscisse No. La plupart des lógiciels de calcul statistique réalisent facilement ces opérations. L'intervalle de confiance est donné par les valeurs extrêmes qui sont les solutions de l'équation du deuxième degré (2) (RICKER, 1975).

$$
N^{2}\left(q^{2}-t^{2} S_{y x} C_{22}\right)-2\left(q^{2} N o-t^{2} S^{2} y x C_{12}\right) N+\left(q^{2} N o^{2}-t^{2} S^{2} y x C_{11}\right)=0
$$

avec :

$$
\begin{aligned}
& \mathrm{C}_{11}=\mathrm{k}\left(\Sigma_{i} \mathrm{Ni}^{2}\right) /\left(\mathrm{k}\left(\Sigma\left(\mathrm{Ni}^{2}\right)-(\Sigma \mathrm{Ni})^{2}\right)\right. \\
& \mathrm{C}_{12}=k\left(\Sigma_{i} \mathrm{Ni}\right) /\left(\mathrm{k}\left(\Sigma\left(\mathrm{Ni}^{2}\right)-(\Sigma \mathrm{Ni})^{2}\right)\right. \\
& \mathrm{C}_{22}=\mathrm{k} /\left(\Sigma\left(\mathrm{Ni}^{2}\right)-\left(\sum \mathrm{Ni}\right)^{2}\right) \\
& \mathrm{T}=\text { la valeur du } \mathrm{t} \text { de Student correspondant à un niveau donné pour } \mathbf{n}-2 \text { degrés de liberté } \\
& \left.S^{2}{ }_{y x}=\left(\sum \mathrm{Ci}^{2}-\left(\sum(\mathrm{Ci} \mathrm{Ni})\right)^{2} / \sum \mathrm{Ni}^{2}\right)\right) / \mathrm{k}-2
\end{aligned}
$$

Dans le cas de notre exemple les résultats sont portés dans le tableau 2. L'intervalle de confiance n'est pas symétrique par rapport à la valeur No estimée.

\begin{tabular}{|l|c|c|c|}
\hline Méthode & de P & Estimation & Intervalle de \\
\hline LESLIE & 0,878 & 115 & confiance \\
\hline DE LURY & 0,859 & 115 & $107-125$ \\
\hline ZIPPIN & 0,871 & 115 & $114-116$ \\
\hline CARLE et & 0,878 & 115 & $115-115$ \\
\hline STRUB & & & \\
\hline
\end{tabular}

Tableau 2 : Estimation de la “population" de truites fario de 10 a $15 \mathrm{~cm}$ de longueur totale. Résultats des différentes méthodes.

Table 2 : Estimates of the size of trout "population" $10-15 \mathrm{~cm}$ total length using different methods. 
b) La méthode de DE LURY

Après la première pêche, il reste No q poissons dans la rivière, après la $k^{\theta}$ pêche il en reste No $q^{k}$ c'est-à-dire :

$$
\text { No }=T /\left(1-q^{k}\right)
$$

Avant la ie pêche, l'effectif présent est égal à No q $\left(^{(}{ }^{-1}\right)$ soit No qi/q. A la ie pêche, l'effectif pêché

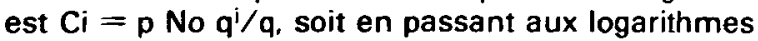

$$
\log \mathrm{Ci}=i \log q+\log \frac{p . \text { No }}{q}
$$

La relation (4) entre $\log \mathrm{Ci}$ et i est donc une droite de pente log q. L'ajustement des points à la droite permet d'estimer log $q$ et donc q puis $p$. No est ensuite calculé par l'équation (3).

La méthode de DE LURY fournit dans le cas de nos deux exemples des résultats proches de ceux obtenus précédemment (tab. 2 et 3 ).

\begin{tabular}{|l|c|c|c|}
\hline Méthode & Estimation & Estimation & Intervalle de \\
de No & confiance
\end{tabular}

Tableau 3 : Estimation de la "population" de truites fario de moins de $10 \mathrm{~cm}$ de longueur totale. Résultats des différentes méthodes.

Table 3 : Estimates of the size of trout "population" $<10 \mathrm{~cm}$ total length using different methods.

\section{5) Les estimations par le maximum de vraisemblance}

\section{a) Méthode de ZIPPIN}

MORAN (1951) a présenté une méthode d'estimation d'effectif de population à partir de résultats de piégeages où les individus capturés n'étaient pas relâchés. ZIPPIN (1956 et 1958) a simplifié cette méthode en fournissant une procédure graphique de calcul et en déterminant la précision asymptotique de l'estimateur. HIGGINS (1985) a publié un programme en Basic qui permet de faire les calculs sans recours aux méthodes graphiques. Les estimations n'en sont que plus exactes. 
Sous les hypothèses présentées en (3), la mëthode emploie le principe du maximum de vraisemblance en considérant la probabilité de retirer $k$ échantillons successifs $C_{1}, C_{2}, C_{3} \ldots C_{k} d^{\prime} u n e$ population de taille No. La probabilité de tirer un seul échantillon de Ci individus à la je pêche est

$$
P(\mathrm{Ci} / \mathrm{ni})=C p^{c i} q^{\mathrm{No}-n i-c i}
$$

C'est le nombre de façons de choisir $\mathrm{Ci}$ individus parmi No-ni.

La probabilité ou vraisemblance de réaliser les captures observées par les $k$ pêches successives est le produit des $k$ probabilités $P(C i)$ noté $P(s)$. On recherche alors la valeur p qui maximise cette probabilité $P(s)$ d'avoir les échantillons observés.

Les équations résultant de ces calculs sont données par ZIPPIN (1956).

$$
\begin{gathered}
R=\frac{\sum_{i=1}^{k}(i-1) C i}{k} \\
i=1 \\
R=\frac{q}{p}-\frac{k q^{k}}{\left(1-q^{k}\right)}
\end{gathered}
$$

R, défini par le rapport de l'équation (6), est utilisé par la méthode graphique de ZIPPIN. Par ordinateur, il est aisé de calculer la valeur de $R$ et ensuite de trouver p par itération avec une précision choisie par le programme. L'estimation de l'effectif de la population est obtenue par:

$$
N=\frac{\sum_{i=1}^{k} C i}{1-q^{k}}
$$

L'erreur standard a pour valeur:

$$
\text { S.E. }{ }^{2}=\frac{N(N-T) T}{T^{2}-N(N-T)(k p)^{2} /(1-p)}
$$

Dans nos deux exemples, le programme de HIGGINS fournit des estimations de 115 et 71 avec des intervalles de confiance moins larges que ceux obtenus avec la méthode de LESLIE (tab. 2 et 3 ).

\section{b) Móthode de CARLE et STRUB}

Dans certains cas les méthodes traditionnelles ne peuvent être employées rigoureusement, car il est évident que la probabilité de capture n'a pas été constante au cours des pêches. L'effectif de la io pêche peut même être supérieur à celui de la (i - 1) $)^{\dot{\theta}}$. CARLE et STRUB (1978) ont développé une méthode nouvelle en pondérant la probabilité de capture $p$ par les deux paramètres d'une fonction de distribution Béta.

Sous cette hypothèse, l'estimation de No est le plus petit entier supérieur ou égal à T qui satisfait l'inégalité suivante:

$$
\begin{aligned}
& N-\frac{N+1}{T+1} \prod_{i}\left[\frac{k N-X-T+\beta+(K-i)}{k N-X+\alpha+\beta+(K-i)} \leq 1\right. \\
& \text { avec } X=\Sigma(k-i) C i \\
& \quad \alpha \text { et } \beta \text { paramètres de la loi Béta. }
\end{aligned}
$$

Il est également facile de calculer $\mathbf{N}$ par itération sur ordinateur. Nous avons écrit un programme calqué sur celui de HIGGINS fournissant cette estimation (voir annexe). La probabilité de capture pest également estimée par maximum de vraisemblance:

$$
P=\frac{T}{k N-X}
$$

La variance de l'estimateur est ensuite calculée par la formule de ZIPPIN.

CARLE et STRUB ont montré que le biais de leur estimation est inférieur au biais de la méthode de ZIPPIN. Ce biais est toujours négatif et toujours faible tant que p reste supérieure à 0.3 (figure 1 ). 


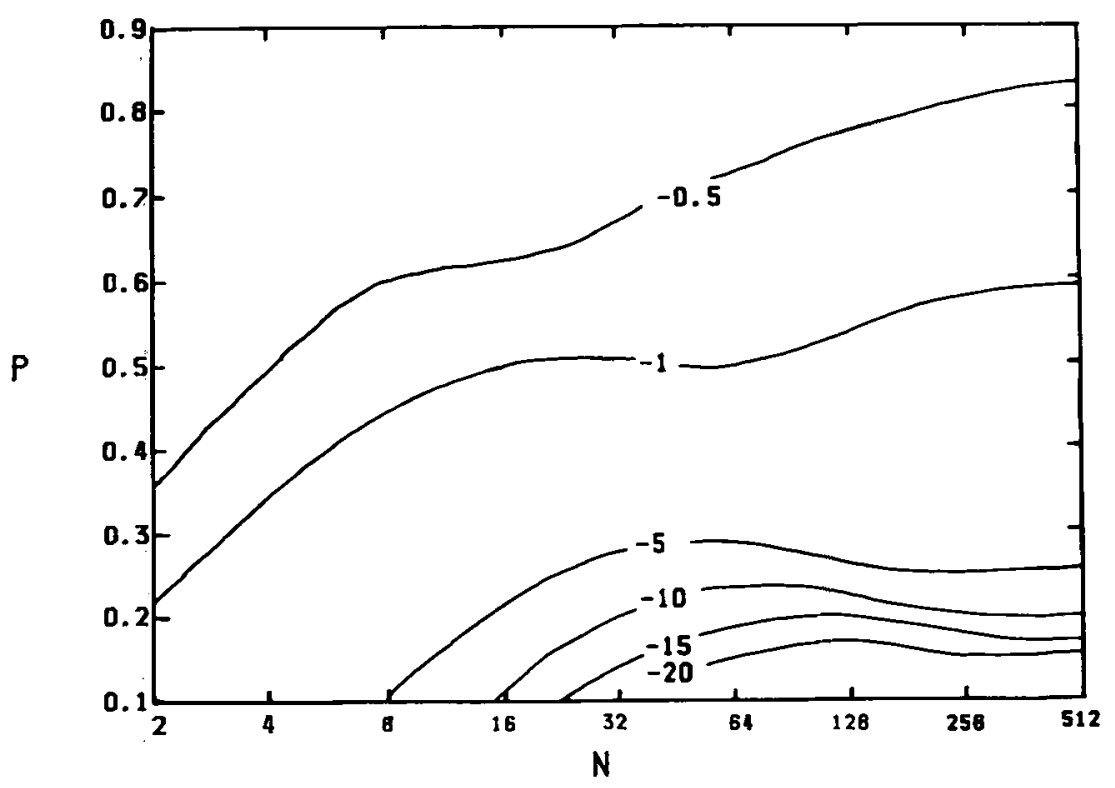

Figure 1 : Biais de l'estimateur de CARLE et STRUB pour des combinaisons de p et $N$, dans le cas de 3 pêches successives (d'après CARLE et STRUB, 1978).

Figure 1 : Biaises of CARLE and STRUB estimates at various combinations of $p$ and $N$, with.K=3 (after CARLE and STRUB, 1978).

\section{CONCLUSIONS}

Le développement de la microinformatique permet d'employer des méthodes nécessitant de nombreux calculs. Les méthodes graphiques employées auparavant introduisaient des erreurs dès la lecture des valeurs sur les graphiques. La programmation de la méthode de ZIPPIN par HIGGINS évite ces erreurs de lecture. Par exemple, COWX (1983) développe un calcul sur 5 pêches successives dont les effectifs sont $72,56,46,30,24$.

Pour $R=1,465$ la valeur de $\left(1-q^{k}\right)$ est lue égale à 0.75 alors que par le calcul par itération on trouve la valeur exacte égale à 0,746 . L'estimation de $\mathrm{N}$ devient 305 alors que COWX fournit 304. De plus les calculs sont très rapides et peuvent être réalisés sur le terrain en temps réel avec un microordinateur portable.

En plus de la satisfaction de faire rapidement des calculs précis, la méthode d'estimation de CARLE et STRUB fournit toujours des intervalles de confiance plus petits que celui calculé par les autres méthodes (Tab. 1 et 3). Les deux cas de pêche successives pris en exemple montrent déjà l'intérêt de la méthode de CARLé et STRUB quand la probabilité de capture p est supérieure à 0,5. Quand cette probabilité est faible, voire croissante d'une pêche à l'autre, toutes les autres méthodes sont mises en échec. CARLE et STRUB (1978) citent le cas d'un chabot Cottus bairdidont les effectifs des captures successives par pêches électriques sont respectivement $\mathrm{C1}=5, \mathrm{C2}=7, \mathrm{C3}=\mathbf{8}$. Dans ce cas, il est possible d'estimer un effectif avec les autres méthodes. La méthode de CARLE et STRUB fournit une estimation de l'effectif de 44 individus et de la probabilité de capture égale à 0,174 . La figure 1 indique qu'une confiance limitée doit être accordée à ce résultat. L'augmentation de l'efficacité de capture vers $p=0,25$ est indispensable.

Seule cette méthode permet d'obtenir ce type de résultats dans tous les cas de pêches successives. Les contraintes imposées aux hypothèses de travail sont plus faciles à satisfaire. Les calculs sont aussi rapides que par la méthode de ZIPPIN. Tous ces arguments conduisent au choix de la méthode de CARLE et STRUB.

\section{REMERCIEMENTS}

L'article présenté ci-dessus entre dans le cadre d'une convention CSP-INRA (83-624 du $6 / 12 / 1983$ ). 


\section{OUVRAGES CITES}

CARLE F.L. and STRUB M.R., 1978 - A new method for estimating population size from removal data. Biometrics, 34, p. $621-630$.

COWX 1.G., 1983 - Review of the methods for estimating fish population size from survey removal data. Fish Manage., 14 (2), p. 67-82.

De LURY D.B., 1947 - On the estimation of biological population. Biometrics, 3, p. 145-147.

HELLAND A., 1913-1914 - Rovdyrene i Norge, Tidskrift for Skogbruk 1913-1914. In: The optimum catch, Hjort et al. (eds.). Hvalradets Skr., 7. p. 92-127.

HIGGINS P.J., 1985 - An interactive computer program for population estimation using the Zipping method. Aquac. Fish. Manage., 1, p. 287-295.

JUNGE C.O. and LIBOSWARSKY J., 1965 - Effects of size selectivity on population estimates based on successive removals with electrical fishing gear. Zool. Listy 14, p. 171-178.

LESLIE P. H. and DAVIS D.H.S., 1939 - An attempt to determine the absolute number of rats in a given area. J. Anim. Ecol., 8, p. 94-113.

MORAN P.A.P., 1951 - A mathematical theory of animal trapping. Biometrika, 38 p. 307-311.

RICKER W.E., 1975 - Computation and interpretation of biological statistics of fish population. Bull. Fish. Res. Board Can., 191, 383 p.

ROBSON D.S. and REGIER H.A., 1968 - Estimation of population number and mortality rates. In: Methods for assessment of fish production in freshwater. Richer (ed.) I.B.P. Handbook, 3; Blackwell, Oxford; p. 124-138.

SEBER G.A.F. and Le CREN E.D., 1967 - Estimating population parameters from catches large relative to the population. J. Anim. Ecol. 36, p. 631-643.

ZIPPIN C., 1956 - An evolution of the removal method of estimating animal populations. Biometrics 12, p. $163-189$.

ZIPPIN C., 1958 - The removal method population and estimation. J. Wildl. Manage., 22, p. 82-90.

\section{ANNEXE}

10 DIM Y (50)

20 PRINT

30 PRINT “ESTIMATION D'UNE POPULATION PAR LA MÉTHODE DE CARLE ET STRUB"

40 CALCUL DE LA CAPTURE TOTALE T

50 PRINT

60 PRINT

70 PRINT "NOM DU SITE DE PÊCHE"

80 INPUT SS

90 PRINT

100 PRINT "DONNEZ LA DATE DE LA PECCHE"

110 INPUT DS

120 PRINT

130 PRINT "DONNEZ LA SURFACE ECHANTILLONNEE EN METTRES CARRES"

140 INPUT A

150 PRINT

160 PRINT "ESPECE ETUDIEE"

170 INPUT FS

180 PRINT

190 PRINT "COMBIEN DE PECCHES SUCCESSIVES?"

200 INPUT $K$

$210 \quad T=0$

$220 \quad S=0$

$230 \quad \mathrm{U}=1$

$240 \quad \mathrm{~N}=0$

250 PRINT

260 PRINT "TAPEZ LE NOMBRE DE POISSONS CAPTURES A CHAQUE PÊCHE"

270 FOR I=1 TO $\mathrm{K}$

280 INPUT $Y(I)$

$290 \quad \mathrm{~T}=\mathrm{T}+\mathrm{Y}(\mathrm{I})$

$300 \quad S=S+(K-1)^{*} Y(I)$

310 NEXT I 
320 SOLUTION DE L'ÉOUATION DE CARLE ET STRUB

$330 \mathrm{M} \$=$ "CARLE et STRUB"

340 FOR $\mathrm{N}=\mathrm{T}$ TO 10000

$350 \mathrm{U}=1$

360 FOR $I=1$ TO $K$

$370 \mathrm{U}=\mathrm{U}^{*}\left(\left(\left(\mathrm{~K}^{*} \mathrm{~N}\right)-\mathrm{S}-\mathrm{T}+1+(\mathrm{K}-1)\right) /\left(\left(\mathrm{K}^{*} \mathrm{~N}\right)-\mathrm{S}+2+(\mathrm{K}-1)\right)\right)$

380 NEXT I

$390 \mathrm{U}=(\mathrm{N}+1)^{*} \mathrm{U} /(\mathrm{N}-\mathrm{T}+1)$

400 IF $U<1$ THEN 420

410 NEXT $N$

420 PRINT " $U<1$ A L'ITÉRATION NUMÉRO", N

$430 \quad \mathrm{D}=\mathrm{T} /(\mathrm{K} * \mathrm{~N}-\mathrm{S})$

$440 \quad$ Q1 $=1-D$

$450 \quad E=2^{*} \operatorname{SOR}\left(N^{*}(N-T)^{*} T /\left(\left(T^{*} T\right)-N^{*}(N-T)^{*}\left(\left(K^{*} D\right)^{*} 2\right) / 01\right)\right)$

460 PRINT

470 PRINT

480 PRINT "RÉSULTATS"

490 PRINT "

500 PRINT

510 PRINT S\$, DS

520 PRINT

530 PRINT "ESPECE DE POISSON ÉTUDIE : “;F\$

540 PRINT "

550 PRINT

560 PRINT "NOMBRE DE POISSONS CAPTURÉS A CHAOUE PECHE"

570 PRINT ".

580 FOR $I=1$ TO K

590 PRINT" ",Y(I)

600 NEXT I

610 PRINT

620 PRINT "AIRE TOTALE ÉCHANTILLONNÉE M2 = ";A

630 PRINT "NOMBRE DE PECHES SUCCESSIVES"; K

640 PRINT "TOTAL DES POISSONS CAPTURES";T

650. PRINT "DENSITÉ MINIMUM PAR METTRE CARRE $=$ ";T/A

660 PRINT “ESTIMATION PAR LA MÉTHODE DE CARLE ET STRUB $\mathrm{N}=$ "; $\mathrm{N}$

670 PRINT "'INTERVALLE DE CONFIANCE A $5 \%=+,-"$ ": E

680. P.RINT "DENSITE ESTIMÉE AU MÉTRE CARRÉ = ";N/A

690 PRINT "INTERVALLE DE CONFIANCE DE LA DENSITE A $5 \%=" ; E / A$

700 PRINT "

710 PRINT

720 PRINT "QUE SOUHAITEZ-VOUS MAINTENANT ?"

730 PRINT "A — UN AUTRE CALCUL SUR LE MÊME SITE, MÊME DATE"

740 PRINT "B - UNE SORTIE DE CES RÉSULTATS SUR IMPRIMANTE"

750 PRINT "D - FIN DE CALCULS"

760 INPUT OS

770 IF O\$ $=$ "A" THEN 160

780 IF $Q \$=$ " $B$ "' THEN 810

790 .IF. OS $=$ "D" THEN 1050

800 GOTO 730

810 LPRINT

820. LPRINT S\$, DS

830 LPRINT

840 LPRINT “ESPECE DE POISSON ÉTUDIÉ;";FS

850 LPRINT “

860 LPRINT

870 LPRINT "NOMBRE DE POISSONS CAPTURES A CHAQUE PÊCHE"

880 LPRINT "

890 FOR $I=1$ TO K

900 LPRINT ". ",Y(I)

910 NEXT I.

920 . LPRINT

$9 \ddot{940}$ LPRINT "AIRE TOTALE ECHANTILLONNÉE M2 = ":A

950 LPRINT "NOMBRE DE PECHES SUCCESSIVES"; $K$

960 LPRINT "TOTAL DES POISSONS CAPTURES";T .

970 LPRINT "DENSITÉ MINIMUM PAR METTRE CARRE $=" ; T /$ A

980. LPRINT "ESTIMATION PAR LA MÉTHODE DE ";MS;" " ";N 
990 LPRINT 'INTERVALLE DE CONFIANCE A $5 \%=+-$ ', E

1000 LPRINT "DENSITE ESTIMEE AU METTRE CARRÉ = N/A

1010 LPRINT “INTERVALLE DE CONFIANCE DE LA DENSITE A $5 \%=“ ; E / A$

1020 LPRINT “"

1030 LPRINT

1040 GOTO 710

1050 END

\section{EXEMPLE D'UNE SORTIE}

RUN

ESTIMATION D'UNE POPULATION PAR LA MÉTHODE DE CARLE ET STRUB NOM DU SITE DE PECHE ? REDON

DONNEZ LA DATE DE LA PÊCHE

? 9/11/1983

DONNEZ LA SURFACE ÉCHANTILLONNÉE EN N.ĖTRES CARRÉS

$? 728$

ESPĖCE ÉTUDIÉE

? TRUITE FARIO < $10 \mathrm{CM}$

COMBIEN DE PECCHES SUCCESSIVES?

? 3

TAPEZ LE NOMBRE DE POISSONS CAPTURÉS A CHAQUE PECHE

$? 40$

$? 18$

? 7

U < 1 A L'ITÉRATION NUMÉRO 69

RESULTATS

REDON $\quad 9 / 11 / 1983$

ESPECCE DE POISSON ÉTUDIÉ : TRUITE FARIO <10 CM

NOMBRE DE POISSONS CAPTURÉS A CHAQUE PEECHE

40

18

7

AIRE TOTALE ÉCHANTILLONNEE M2 $=728$

NOMBRE DE PECHES SUCCESSIVES 3

TOTAL DES POISSONS CAPTURÉS 65

DENSITÉ MINIMUM PAR MËTRE CARRÉ $=8.928572 \mathrm{E}-02$

ESTIMATION PAR LA MÉTHODE DE CARLE ET STRUB $\mathrm{N}=69$

INTERVALLE DE CONFIANCE A $5 \%=+,-5.935722$

DENSITÉ ESTIMEEE AU MĖTRE CARRÉ $=9.478022 \mathrm{E}-02$

INTERVALLE DE CONFIANCE DE LA DENSITÉ A $5 \%=8.153464 E-03$

QUE SOUHAITEZ-VOUS MAINTENANT?

A - UN AUTRE CALCUL SUR LE MEME SITE, MEME DATE

B - UNE SORTIE DE CES RÉSULTATS SUR IMPRIMANTE

D - FIN DES CALCULS

? D

ok 